\title{
DYNAMIC BEHAVIOR OF A LIQUID ROCKET ENGINE FEEDING SYSTEM
}

\author{
Tamer N. MAHMOUD*, M. Galal RABIE**, M. Allam EL-SENBAWI***
}

\begin{abstract}
This paper is the second part of a study conducted to investigate the dynamic behavior of a liquid propellant rocket engine. It is dedicated to complete the modeling, simulation and analysis of the dynamic behavior of the feeding system of a typical rocket engine. The elements involved are the gas generator, the turbo-pump assembly, and the combustion chamber. These individual elements are treated as separate units before dealing with the integrated system. The system incorporates the combustion chamber controller, the thrust controller, the gas generator controller, and the safety valve. Non-linear mathematical models and empirical relations have been deduced and used to develop computer simulation programs using MATLAB SIMULINK technique. The study was extended to investigate the static and dynamic behaviors of such components. The study showed that the thrust reaches always a new steady state value corresponding to pre-compression distance of the thrust controller spring and through regulating the combustion chamber pressure. The system, which has two power ratings, operates at a stable, self-regulated condition with slightly under-damped nature of settling time in switching from first to second power rating. The developed simulation programs could help investigate and analyze other similar liquid propellant rocket engines.
\end{abstract}

\section{KEY WORDS}

Rocket propellant feeding system, liquid rocket engine, static behavior, dynamic behavior, liquid propellant engine control, computer simulation, SIMULINK.

\footnotetext{
* $\quad$ Major Eng., Egyptian Armed Forces.

** Professor, Modern Academy for Engineering \& Technology, Cairo, Egypt.

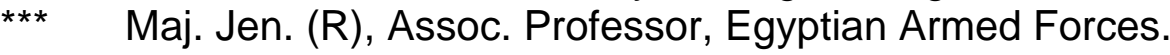




\section{INTRODUCTION}

The simulation by digital computers plays an important role in the development and analysis of behavior of hydraulic control systems. It allows observing the variations of the operational parameters in the transient conditions. Moreover, it provides a powerful tool for the optimization of the system constructional parameters and may help propose a new system with a programmed thrust-time relation.

This paper deals with the behavior of the feeding system of a liquid propellant rocket linked to the engine assembly that comprises a gas generator; a pump drive assembly, and a combustion chamber. The system involves a set of valves controlling the pressure and flow rate of fuel and oxidizer. The valves have peculiar design imposed by the constraint that these two liquids should never come into contact outside the gas generator or the combustion chamber. Actually, it is almost impossible to find any similar study in the available literature. However, the library is rich with papers dealing with the classical, turbo-pump, and electrohydraulic systems. An exception was the work of Rabie ${ }^{[1]}$, which dealt theoretically with the dynamic behavior of a pilot operated pressure reducer. Rabie investigated also the same valve performance ${ }^{[2]}$ by developing a black bond graph model. However, the study of publications of Margolis ${ }^{[3]}$, Furst ${ }^{[4]}$, Akers ${ }^{[5]}$, Stepanoff ${ }^{[6]}$, and Merrit ${ }^{[7]}$ gave good support to the theoretical part of the present paper.

A schematic of the investigated system is shown in Figs.1, 2 and 3. Interested readers are directed to the work of $\operatorname{Tamer}^{[8]}$. for more detailed description of the system, which involves a combustion chamber controller, a gas generator controller, a thrust controller, a safety valve, a gas generator, a pump drive assembly, and a combustion chamber. The study involves system description, deduction of nonlinear mathematical model, empirical relations, and the development of a computer simulation program. The simulation is carried out using the SIMULINK program. Effect of some operational and constructional parameters on the static and dynamic behavior of the system is investigated.

\section{THE ROCKET MOTOR}

\subsection{Technical Description}

The rocket motor under study consists of feeding and control assemblies and engine assemblies. The system, shown in Fig.2, encompasses the following elements. The combustion chamber controller (1) The gas generator combustion chamber is fed by fuel and oxidizer through the thrust controller (2) and the gas generator controller (3). The thrust controller maintains the required pressure in the combustion chamber at the first and second power ratings. This controller carries out the engine change over from first-to-second power rating. Safety valve (4). The combustion products are guided to drive the gas turbine. The pump drive assembly consists of gas generator (5), high speed turbine (6), fuel pump (7), and oxidizer pump (8). The combustion chamber (9), converts chemical energy stored in the propellants into thermal energy of combustion gases. These gases are then accelerated through the exhaust nozzle producing thrust. Oxidizer, Fuel inlet valve: Separate suction cavity of oxidizer and fuel pumps from oxidizer and fuel tanks till the moment of engine starting. Powder starter: Produces gases to rotate the turbine of the pump drive assembly at the moment of the engine starting, Fig.1. 


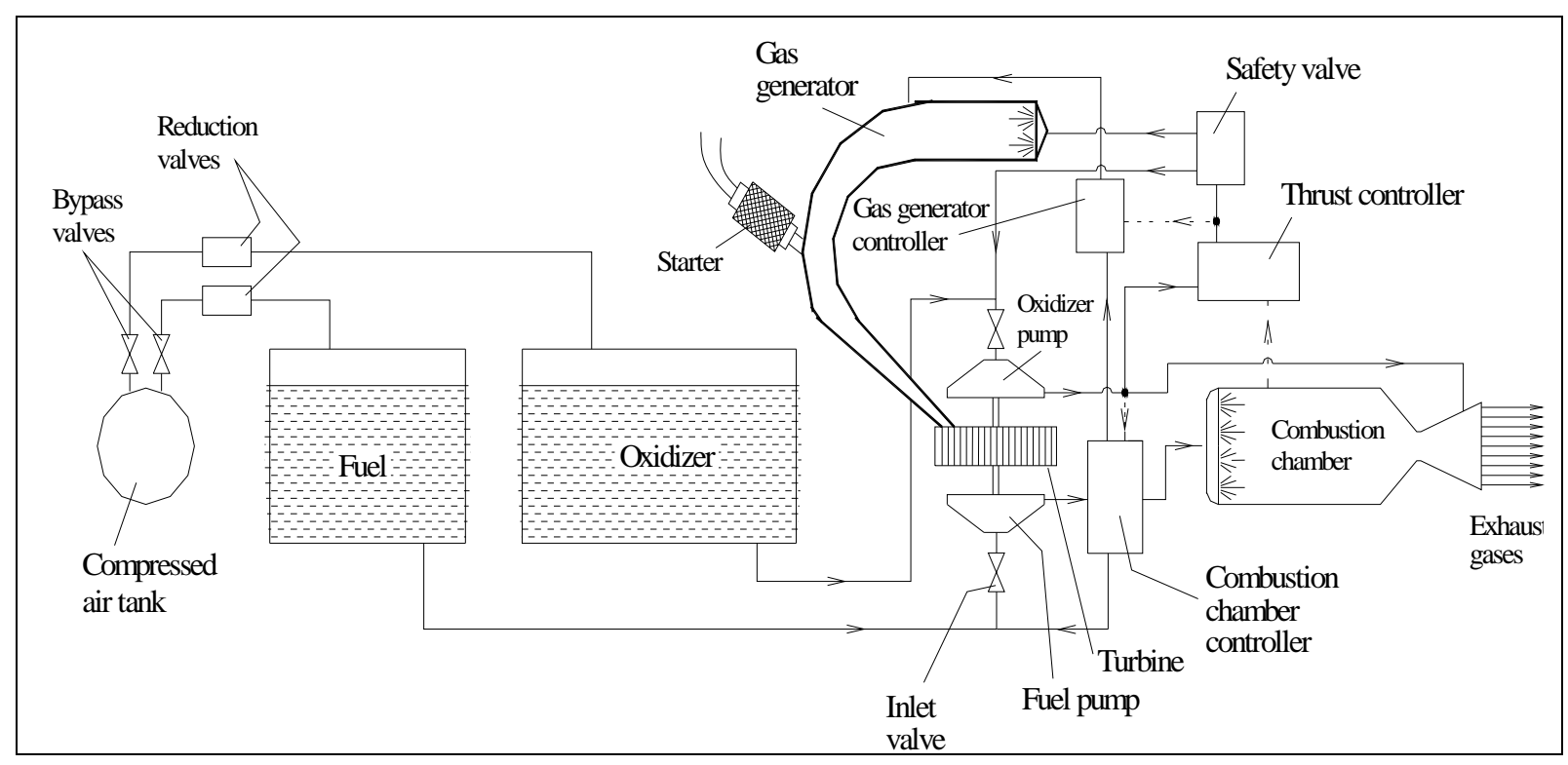

Fig.1. Schematic of the rocket engine assemblies

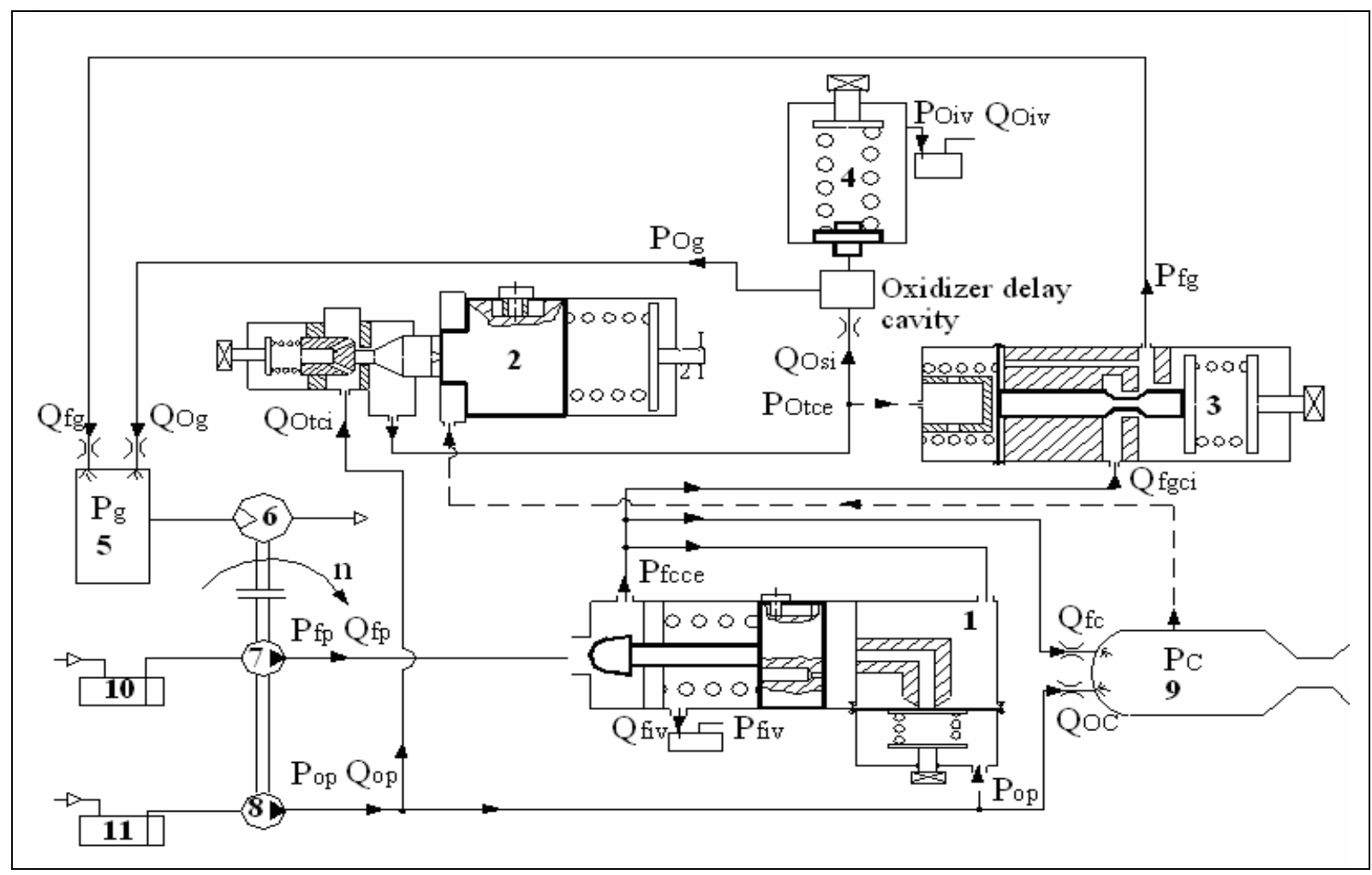

Fig.2. Block diagram of the rocket engine assemblies 


\subsection{Principle of Operation}

An electric pulse is fed to powder rocket booster that operates and the rocket is launched. The powder starter operate and the combustion products coming to the turbine (6), Fig.2. The assembly rotor starts and the combustion products are evacuated through exhaust pipe. Oxidizer and fuel fed into the combustion chamber and gas generator come in contact and ignite spontaneously. After the starter powder charge stops burning, the rotation of the pump drive assembly is maintained by the combustion products coming from the gas generator. The starting processes are over and the sustainer gains the first power rating. The powder rocket booster is separated from the rocket after 1.4 second.

\section{A- Operation of sustainer at first rating}

The first rating of sustainer operation is insured by corresponding adjustment of all assemblies of the engine and is maintained by control valves. When the pressure in the combustion chamber deviates from a preset value, increase for instance, the thrust controller increase the hydraulic resistance of the line delivering oxidizer to the gas generator. This reduces consumption of oxidizer and fuel in the gas generator. The pressure in the gas generator falls; the turbine power decreases; the rotor of the pump drive assembly slows down; the pressure of the propellant components at the outlet of the pumps is reduced; and consequently the pressure in the combustion chamber goes back to the normal value.

It is necessary to control the combustion chamber pressure to secure high accuracy of time required to accelerate the rocket to the cruising speed. When fuel pressure at the combustion chamber inlet tends to exceed the oxidizer pressure, combustion chamber controller offers high hydraulic resistance in the fuel pipeline, causing the fuel pressure at the combustion chamber inlet to decrease to the oxidizer pressure.

Securing equal pressures of propellant components at the combustion chamber inlet is necessary to ensure high reliability of combustion chamber function from one part to reduce additional propellant reserve in tanks. The purpose of the gas generator controller is similar to that of the combustion chamber controller.

\section{B- Operation of sustainer at second rating}

As soon as the rocket gains the cruising speed, an electric pulse to the squibs of the thrust controller comes from the speed pickup causing corresponding changes in the controller adjustment. The power rating of the engine is changed accordingly. This involves increase of hydraulic resistance in the line feeding the gas generator with oxidizer and, therefore, decrease of oxidizer consumption through the gas generator causes the fuel consumption to reduce; the pressure in the gas generator goes down, the mass flow rate of gases used to rotate the rotor of the pump drive assembly reduces. The direct result is lower propellant mass flow rates, lower pressure at of combustion down to the preset value. Now the engine operates at the second rating.

\section{THE FEEDING ASSEMBLY}

The feeding assembly consists of pump drive assembly, gas generator, oxidizer inlet, fuel inlet, powder starter, and exhaust pipe. Figure 3 shows the scheme and block diagram of the feeding assembly. Figure 4 shows the block diagram of the pumping assembly. In this study, the function of the gas generator, gas turbine, fuel pump, and oxidizer pump will be described by empirical relations that have been deduced based on the manufacturer data. 


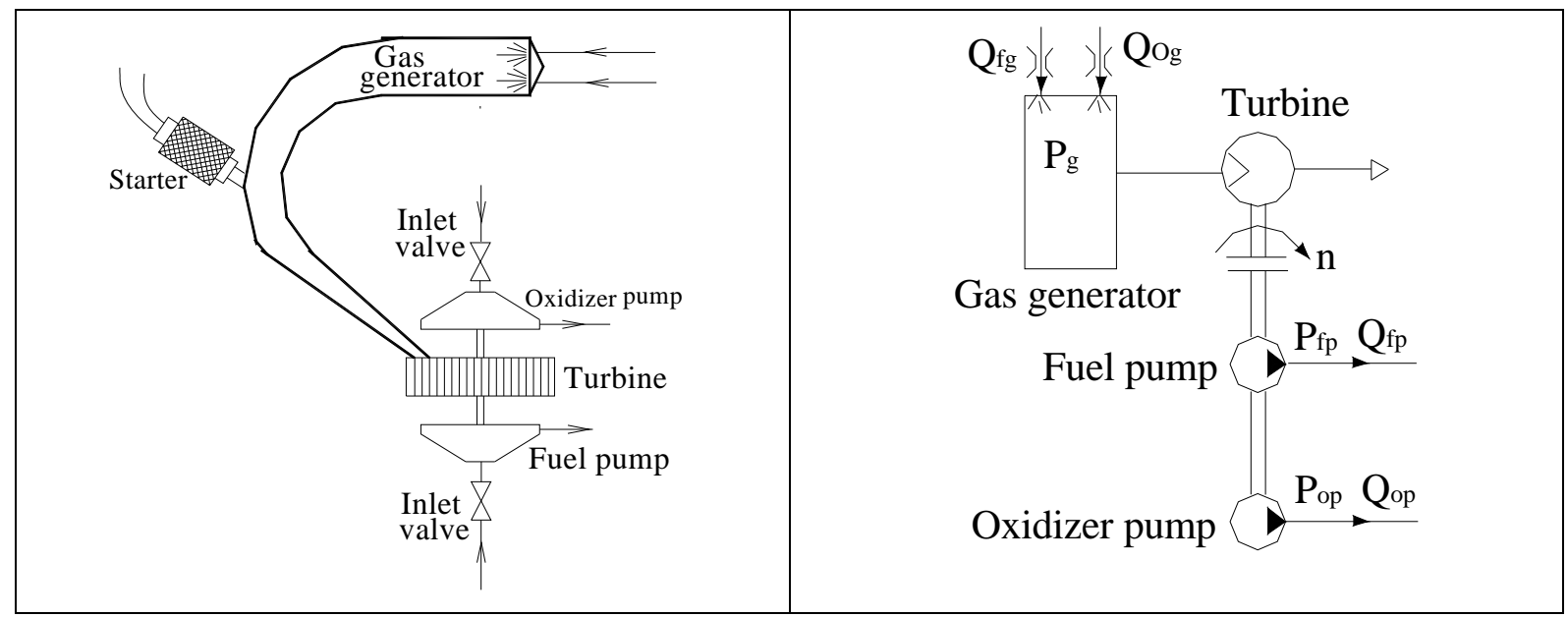

Fig.3. Scheme and block diagram of the feeding assembly

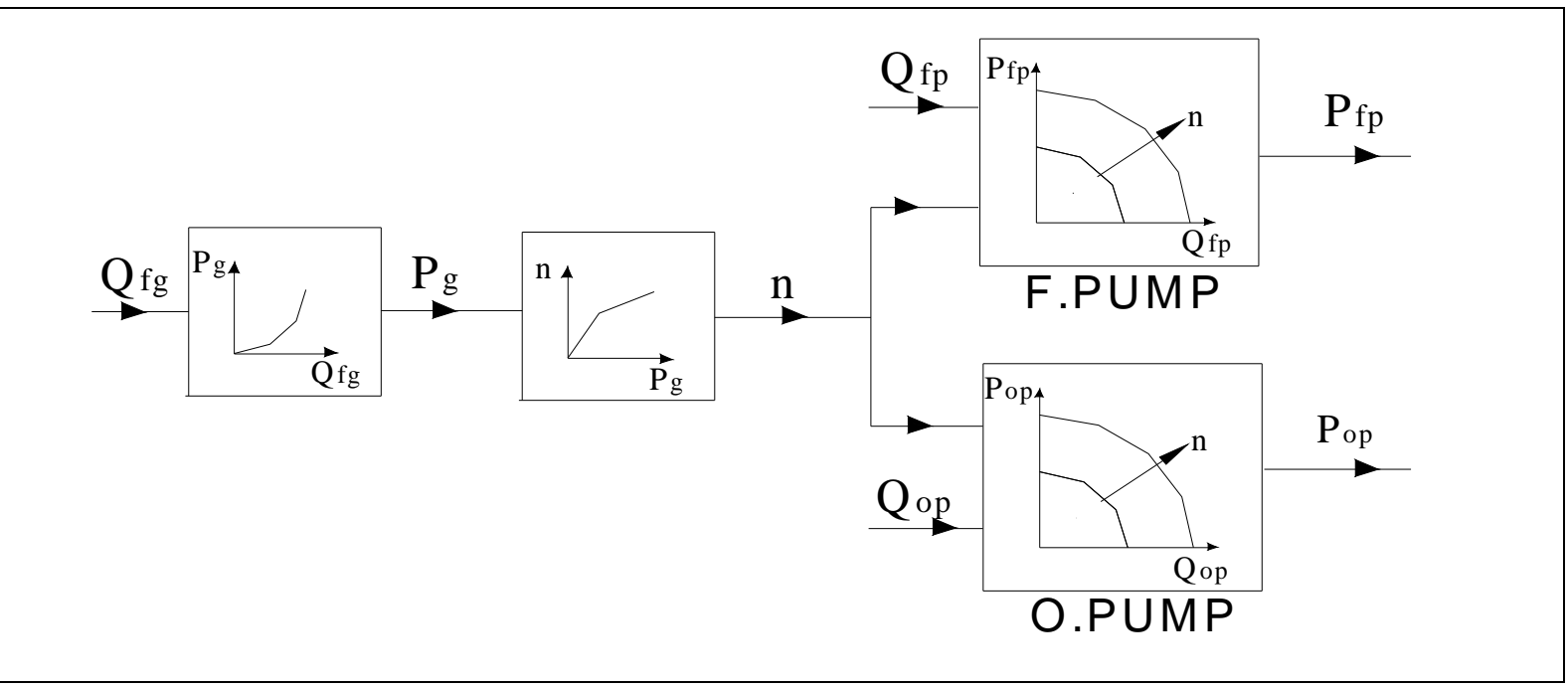

Fig.4. Block diagram of the pumping assembly

\subsection{Gas Generator}

\section{A. Construction and operation}

The gas generator is a non-detachable welded structure consisting of a head, a chamber and a connector. The main elements of the head are the cap, oxidizer injectors, fuel injectors, and filters. The filter is protecting the oxidizer injectors (worm, centrifugal type) from choking.

Oxidizer is fed to the gas generator through pipe connector, filter, and is injected to the chamber through oxidizer injectors. Fuel is fed to the gas generator through cooling space (formed by cylinder and cylinder jacket), filter, and is injected to the chamber through fuel injectors. 
In the gas generator chamber, as oxidizer and fuel come in contact, they ignite spontaneously and the combustion products are delivered to the pump drive assembly through the exhaust branch pipe.

\section{B. Mathematical model}

The gas generator characteristics are studied taking into consideration the flow rate of fuel as a known propellant component. The fuel flow from gas generator controller to the gas generator has the following values from manufacturing data:

$$
\mathrm{Q}_{\mathrm{fg}}\left(1^{\text {st }} \text { rating }\right)=0.00063 \mathrm{~m}^{3} / \mathrm{s}, \quad \mathrm{Q}_{\mathrm{fg}}\left(2^{\text {nd }} \text { rating }\right)=0.000559 \mathrm{~m}^{3} / \mathrm{s}
$$

The fuel and oxidizer are injected to the gas generator with a certain mixture ratio to produce combustion gases. The combustion gas pressure, [bar] is function of the fuel flow rate, $\left[\mathrm{m}^{3} / \mathrm{s}\right]$ according to the following empirical relation which based on the simulation program results.

$$
P_{g}=5.7 * 10^{35}\left(Q_{f g}\right) 9.084
$$

The gas generator characteristics, calculated by using empirical relations, together with matched manufacturing data are plotted in, Fig.5.

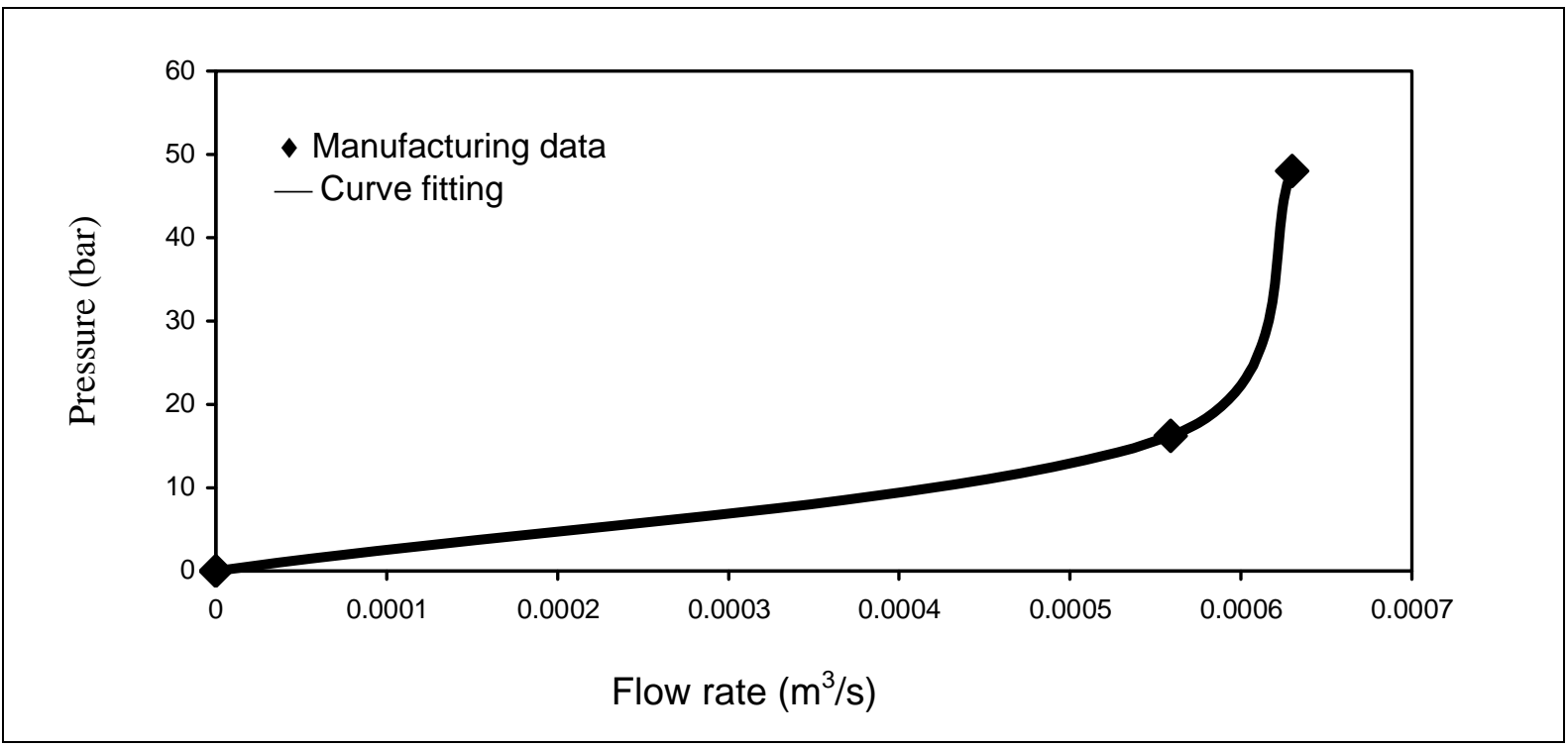

Fig.5. Gas generator characteristics

\subsection{Pump Drive Assembly}

\section{A. Construction and operation}

The pump drive assembly serves to create required pressure of propellant components at the inlet of the combustion chamber and the gas generator. The pump drive assembly consists essentially of a gas turbine, an oxidizer pump and a fuel pump. The case of the pump drive assembly is composed of two halves, a casing of fuel pump and a casing of oxidizer pump, secured to each other with studs. Copper gasket is installed between flanges of oxidizer and fuel pump casings to provide adequate sealing. 
Each of the oxidizer and fuel pump wheels consists of vane wheel and cover soldered to each other, the vane wheel of the oxidizer pump has 6 vanes while the vane wheel of the fuel pump has 12 vanes. The rotor of the gas turbine is composed of disc made integral with the shaft and vanes welded to disc. The rotor has 70 vanes.

When starting the engine, the turbine rotor is rotated by the action of gases from the powder starter on the turbine vanes, then by gases from the gas generator. Exhaust gases are evacuated through the exhaust pipe.

Both oxidizer and fuel pumps are of centrifugal type. Oxidizer from the wheel of the pump runs into the spiral casing from where it is delivered into the combustion chamber and the gas generator. Fuel from the wheel of the pump runs into the spiral casing from where it is delivered to combustion chamber controller.

\section{B. Mathematical model and simulation}

\subsection{Turbine}

The gas generator combustion gases cause the turbine rotation at a certain speed depending on the gas pressure according to the following empirical relation which based on the simulation program results.:

$$
\mathrm{n}_{\text {Turbine }}=0.51767 \times\left(\mathrm{P}_{\mathrm{g}}\right)^{0.42689}
$$

The turbine characteristics, calculated by using empirical relations, are plotted in, Fig.6, That carries the manufacturer data which are well matched with the model.

\subsection{Fuel pump}

The operating pressures (input, output), speeds, and flow rates of the fuel pump, given by the manufacturer, are summarized in Table 1.

\section{Table 1. Fuel pump data}

\begin{tabular}{|ccccc|}
\hline \hline Modes & $\mathrm{n}_{\mathrm{fp}}$ [r.p.m] & $\mathrm{P}_{\mathrm{in}}[\mathrm{bar}]$ & $\mathrm{P}_{\mathrm{out}}[\mathrm{bar}]$ & $\mathrm{Q}_{\mathrm{fp}}\left[\mathrm{m}^{3} / \mathrm{s}\right]$ \\
\hline \hline Out of operation & 0 & 0 & 0 & 0 \\
First rating & 22100 & 2.5 & 88.5 & $1.48 \times 10^{-3}$ \\
Second rating & 13900 & 3 & 35 & $9.69 \times 10^{-4}$ \\
\hline \hline
\end{tabular}

The function of the fuel pump is described by:

$$
\begin{aligned}
& P_{f p}=\left(a_{2}\right)-\left(b_{2} \times Q_{f p}^{2}\right) \\
& a_{2}=\psi \frac{u_{f p}^{2}}{g} \rho_{f} g=\psi u_{f p}^{2} \rho_{f}=\psi\left(\pi D_{f} n_{f p}\right)^{2} \rho_{f}=182.09 n_{f p}^{2} \\
& b_{2}=260.62 \times 10^{10} n_{f p}^{0.173}
\end{aligned}
$$

\begin{tabular}{|c|c|c|}
\hline$a_{2}$ & Fuel pump shut-off pr & essure \\
\hline$D_{f}$ & Fuel pump diameter & $=0.15$ \\
\hline$\psi$ & Constant & $=0.585$ \\
\hline & Fuel denisty & $=820$ \\
\hline
\end{tabular}

Where 
These characteristics, together with manufacturing data are shown in Fig. 7.

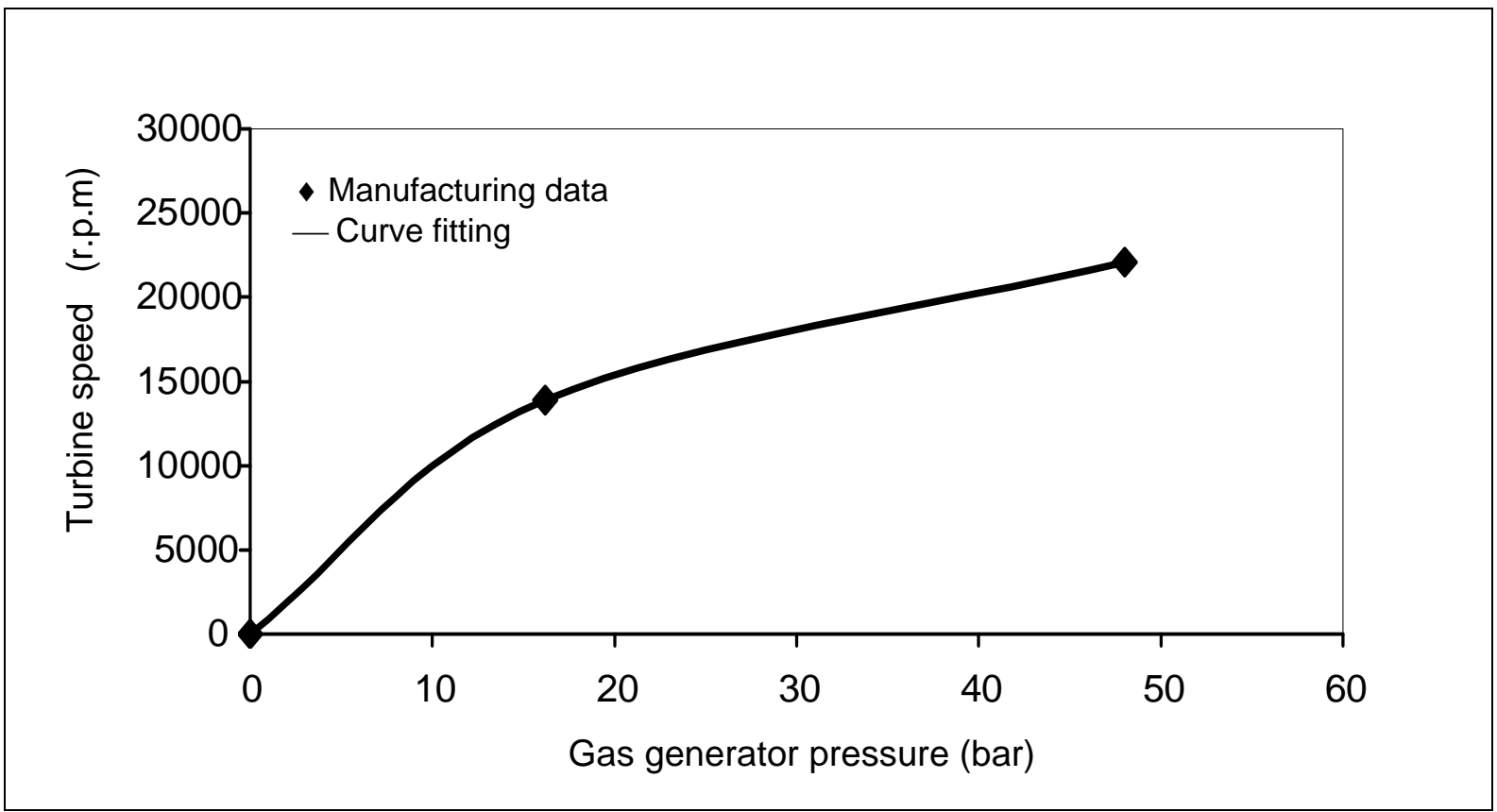

Fig.6. Turbine characteristics

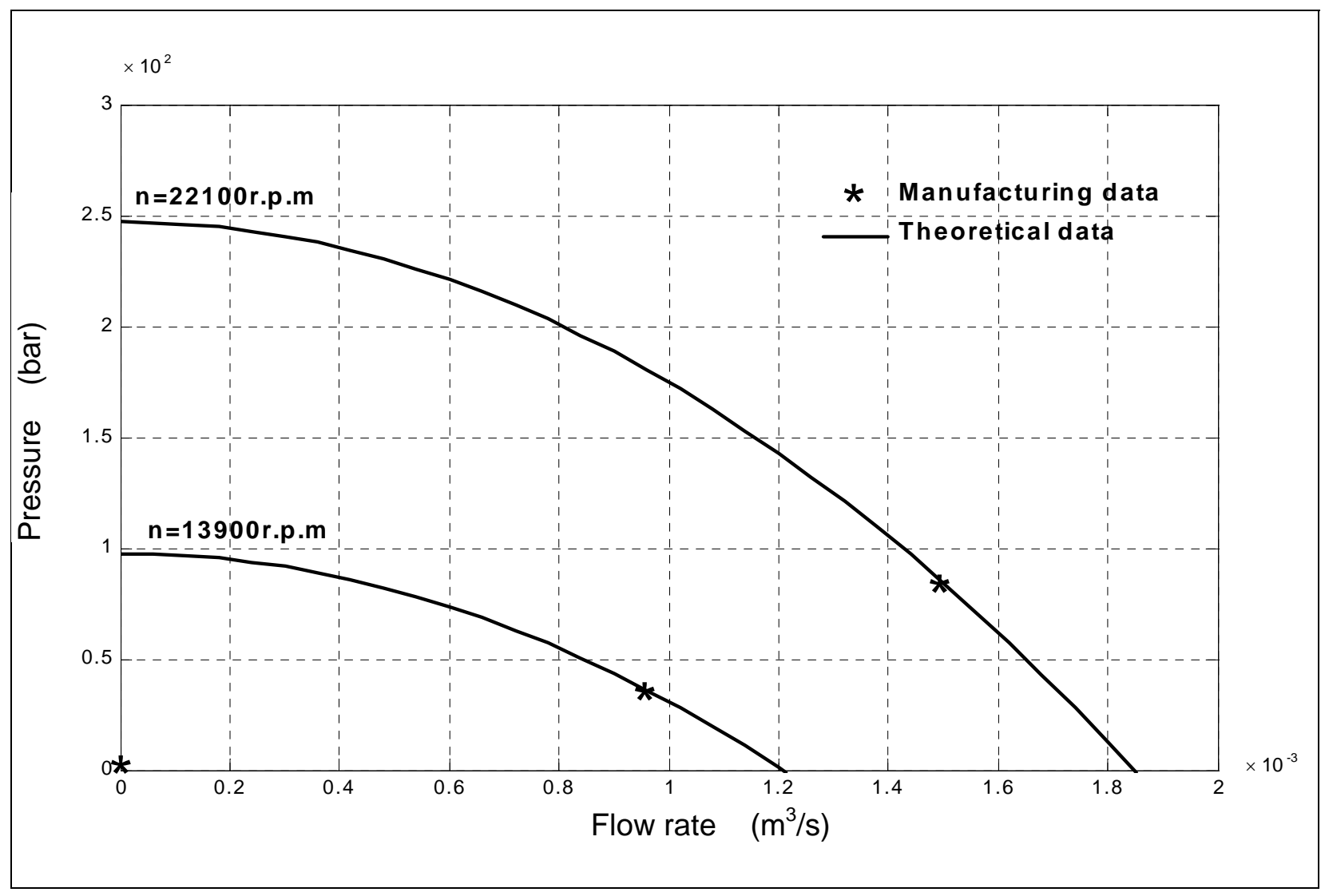

Fig.7. Fuel pump characteristics 


\subsection{Oxidizer pump}

The operating pressures (input,output), speed, and flow rates of the oxidizer pump, given by the manufacturer, are summarized in Table 2.

\section{Table 2. Oxidizer pump data}

\begin{tabular}{|ccccc|}
\hline Modes & $\mathrm{n}_{\text {op }}[$ r.p.m] & $\mathrm{P}_{\text {in }}[\mathrm{bar}]$ & $\mathrm{P}_{\text {out }}[\mathrm{bar}]$ & $\mathrm{Q}_{\text {op }}\left[\mathrm{m}^{3} / \mathrm{s}\right]$ \\
\hline Out of operation & 0 & 0 & 0 & 0 \\
First rating & 22100 & $0.025 \times 10^{7}$ & $0.81^{\star} 10^{7}$ & $2.35^{\star} 10^{-3}$ \\
Second rating & 13900 & $0.03 \times 10^{7}$ & $0.318^{\star} 10^{7}$ & $1.54^{\star} 10^{-3}$ \\
\hline \hline
\end{tabular}

The function of the fuel pump is described by:

$$
\begin{aligned}
\mathrm{P}_{\mathrm{op}} & =\left(\mathrm{a}_{1}\right)-\left(\mathrm{b}_{1} * \mathrm{Q}_{\mathrm{op}}{ }^{2}\right) \\
\mathrm{a}_{1} & =\left(\left(\frac{\mathrm{u}_{\mathrm{op}}{ }^{2}}{\mathrm{~g}}\right)\right)\left(\rho_{\mathrm{o}} \mathrm{g}\right) \Psi=\mathrm{u}_{\mathrm{op}}{ }^{2} \rho_{\mathrm{o}} \Psi=\left(\pi \mathrm{D}_{\mathrm{op}} \mathrm{n}_{\mathrm{op}}\right)^{2} \rho_{\mathrm{o}} \Psi=224.553\left(\mathrm{n}_{\mathrm{op}}\right)^{2} \Psi \\
\mathrm{b}_{1} & =1.4766 * 10^{12}\left(\mathrm{n}_{\mathrm{op}}\right)^{0.17}
\end{aligned}
$$

where $D_{\text {op }}$ Oxidizer pump diameter $=0.12 \mathrm{~m}$
$\rho_{0} \quad$ Oxidizer density
$=1580 \mathrm{Kg} / \mathrm{m}^{3}$
$\mathrm{a}_{1} \quad$ Oxidizer pump shut-off pressure $\mathrm{Pa}$

Oxidizer pump characteristics, together with manufacturer data are shown in Fig. 8.

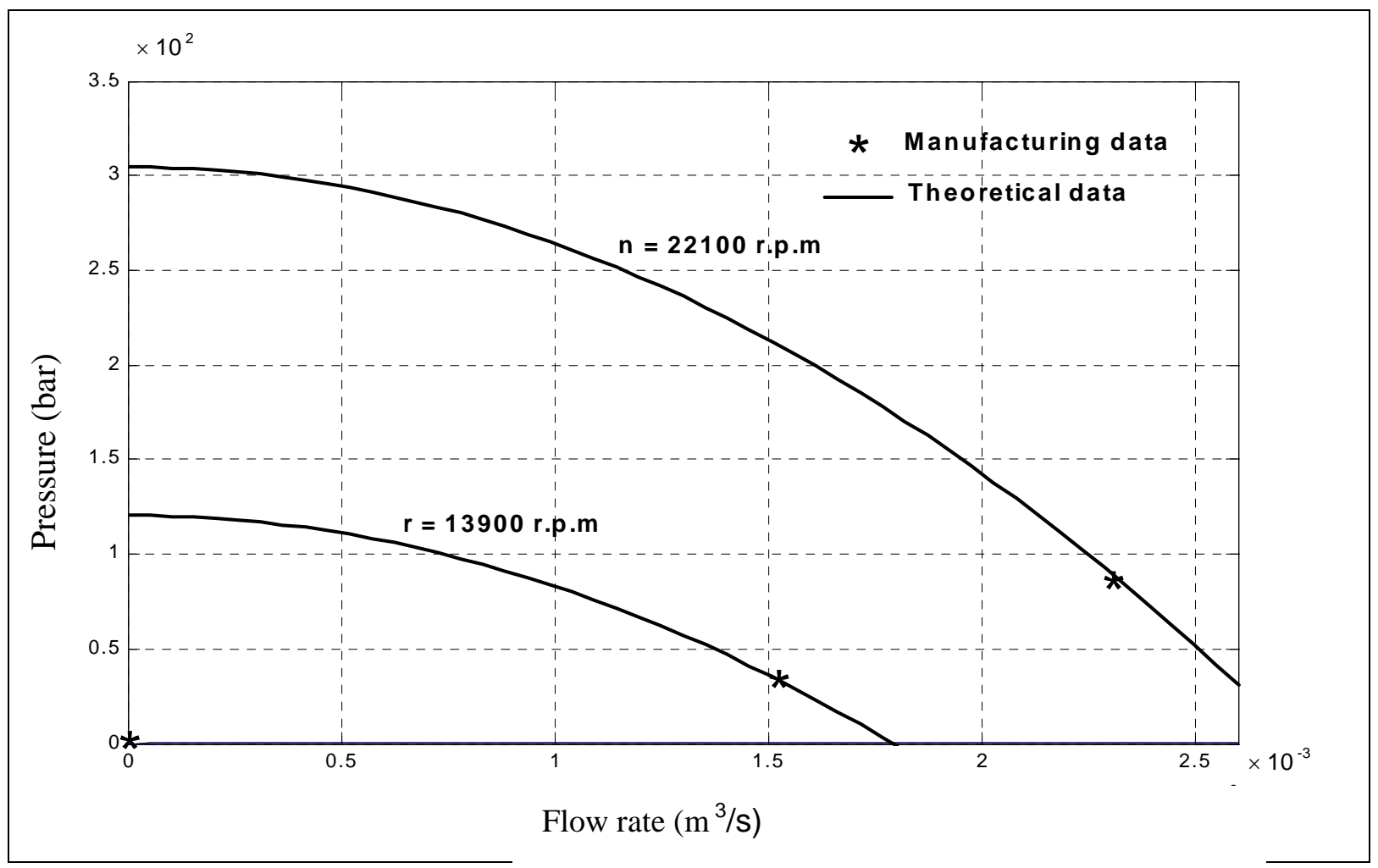

Fig.8. Oxidizer pump characteristics 


\section{COMBUSTION CHAMBER}

\subsection{Construction and Operation}

The combustion chamber is welded to the engine frame. The combustion chamber has a solid welded construction consisting of three essential parts: the head, the chamber, and the nozzle. The head is composed of three caps (making up the oxidizer and fuel cavities), injectors, a spray sheet ring, connectors and filters. Fuel and oxidizer inlet cavities are provided with filtering screens. The number of connectors is three for connecting chamber cavities with thrust controller, measuring the pressure in the chamber, and measuring the pressure before fuel and oxidizer injectors. The combustion chamber is a cylinder with convergent section. Soldered to nozzle ring, a closure is provided to make the engine airtight while storing the rocket. The closure has welded connector for engine pressure test when preparing the sustainer for operation. The space between inner and outer shells along the entire length of the combustion chamber is $2 \mathrm{~mm}$.

Oxidizer is fed to the combustion chamber through two inlets; throttle plates, connector, cooling space, filter and oxidizer injectors. Fuel is supplied to the combustion chamber through pipe connector; throttle plate, pipe, filter, and fuel injectors. A part of fuel coming into combustion chamber holes in the spray sheet ring forms near wall of the chamber a layer of gas enriched with fuel and having somewhat lowered temperature. The gas layer protects inner surface of cylinder and nozzle from burning through. In the combustion chamber fuel and oxidizer burn out. Combustion products flow out through the nozzle.

At the engine start, the propellant components ignite spontaneously as they come in contact; the closure is broken off and thrown away by exhaust gases.

\subsection{Mathematical Model}

The combustion chamber characteristics are studied by taking into consideration the flow rate of one feeding propellant components (fuel). The fuel flows from combustion chamber controller to the combustion chamber at the following flow rates according to the manufacturing data:

$$
\mathrm{Q}_{\mathrm{fc}}\left(1^{\text {st }} \text { rating }\right)=8.49 \times 10^{-4} \mathrm{~m}^{3} / \mathrm{s}, \quad \mathrm{Q}_{\mathrm{fc}}\left(2^{\text {nd }} \text { rating }\right)=4.09 \times 10^{-4} \mathrm{~m}^{3} / \mathrm{s}
$$

The fuel and oxidizer injected to the combustion chamber are burned with certain mixture ratio to produce combustion gases. The combustion gas pressure can be expressed as function of the fuel flow rate according to the following empirical relation which based on the simulation program results:

$$
\mathrm{P}_{\mathrm{c}}=4.8 \times 10^{9}\left(\mathrm{Q}_{\mathrm{fc}}\right)^{0.97}
$$

The combustion gases are exhausted from the nozzle with high speed resulting in the rocket thrust. The rocket thrust can be expressed as a function of combustion pressure according to the following empirical relation which based on the simulation program results:

$$
\mathrm{T}=4.8 \times 10^{-4}\left(\mathrm{P}_{\mathrm{c}}\right)^{1.1}
$$




\section{THRUST MAGNITUDE CONTROL}

In many situations it would be required to modify the thrust magnitude depending on the mission allocated to the missile. Since the rocket thrust $(T)$ is a function of the flow rates of the fuel and oxidizer, it could be modulated through adjusting their pressures. These pressures are controlled by the pre-compression distance of the thrust controller spring, $\left(\mathrm{X}_{\mathrm{o} 2}\right)$, Fig.10.

During the first rating, the spring pre-compression is maximum. The rocket switches to the second rating by allowing this spring to expand. Therefore, the fuel and oxidizer pressures and consequently the thrust, are controlled by the spring precompression distance of the thrust controller. command current. The transient response of the thrust to step variations of the spring pre-compression was calculated. The calculation results of the thrust response to single step variation of spring pre-compression from first to second power rating and multiple step variations are shown in, Fig. 11 \& Fig, 12., respectively. Using an electro-hydraulic servo actuator can control the spring seat position. In this case the command current controls the spring pre-compression and hence the operating mode of the rocket.

The calculations were carried out keeping all operational and constructional parameters of the integrated feeding system constant. The results show that the thrust reaches always a new steady state value corresponding to the spring precompression distance of the thrust controller. For a step up to pre-compression the response is over damped with settling time within $10 \mathrm{~ms}$. For a step down precompression the response is slightly under damped with settling time within $5 \mathrm{~ms}$.

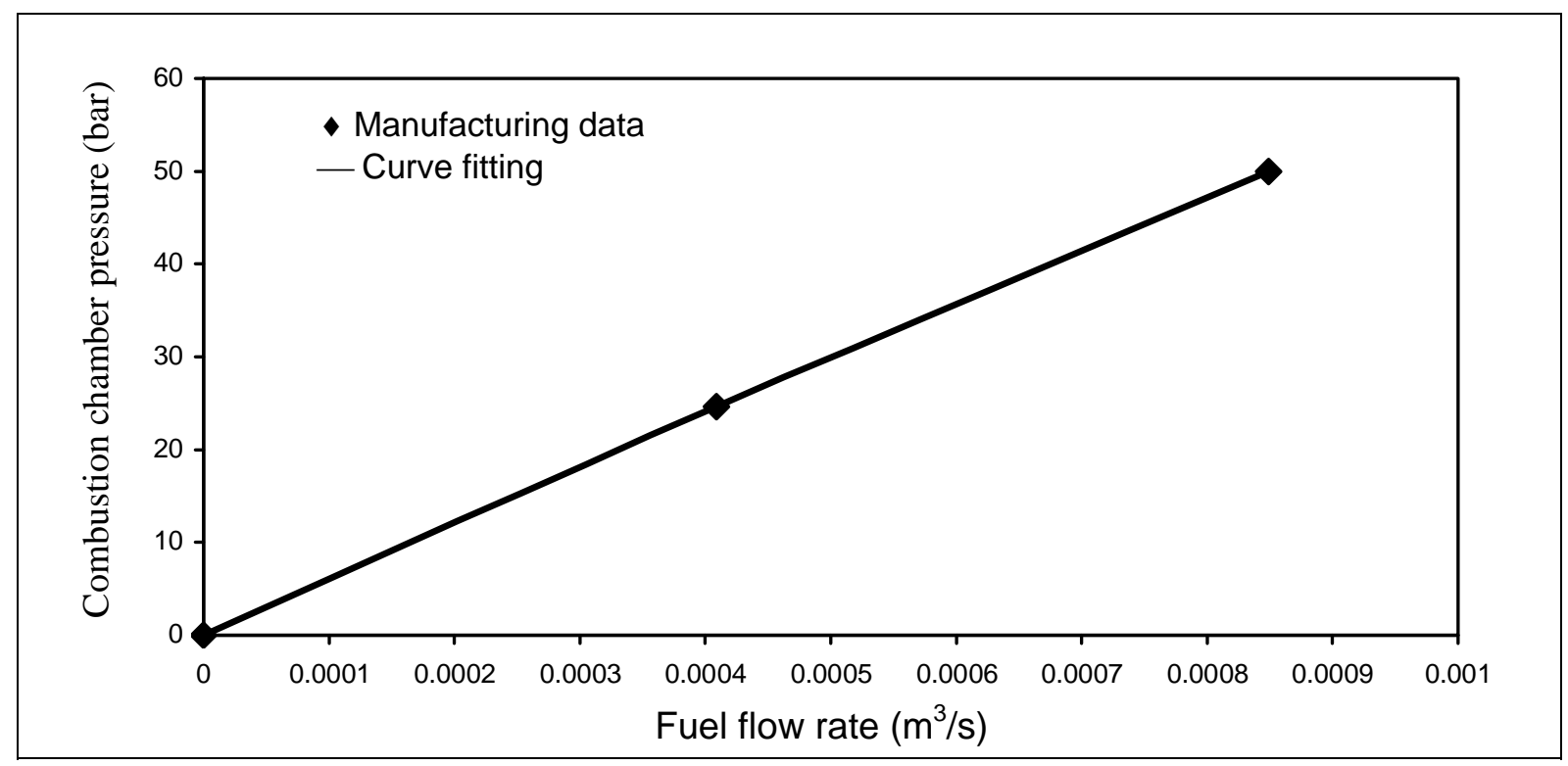

Fig.9. Combustion chamber characteristics 


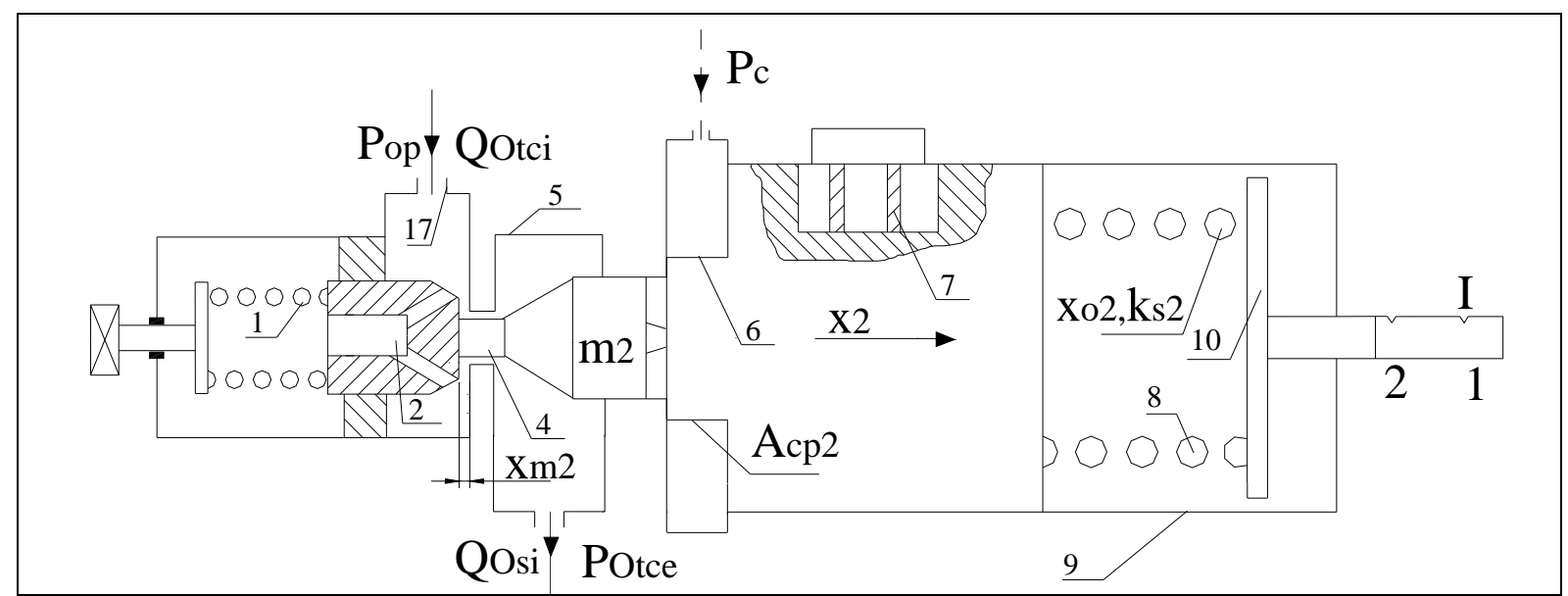

Fig.10. Scheme of the thrust controller

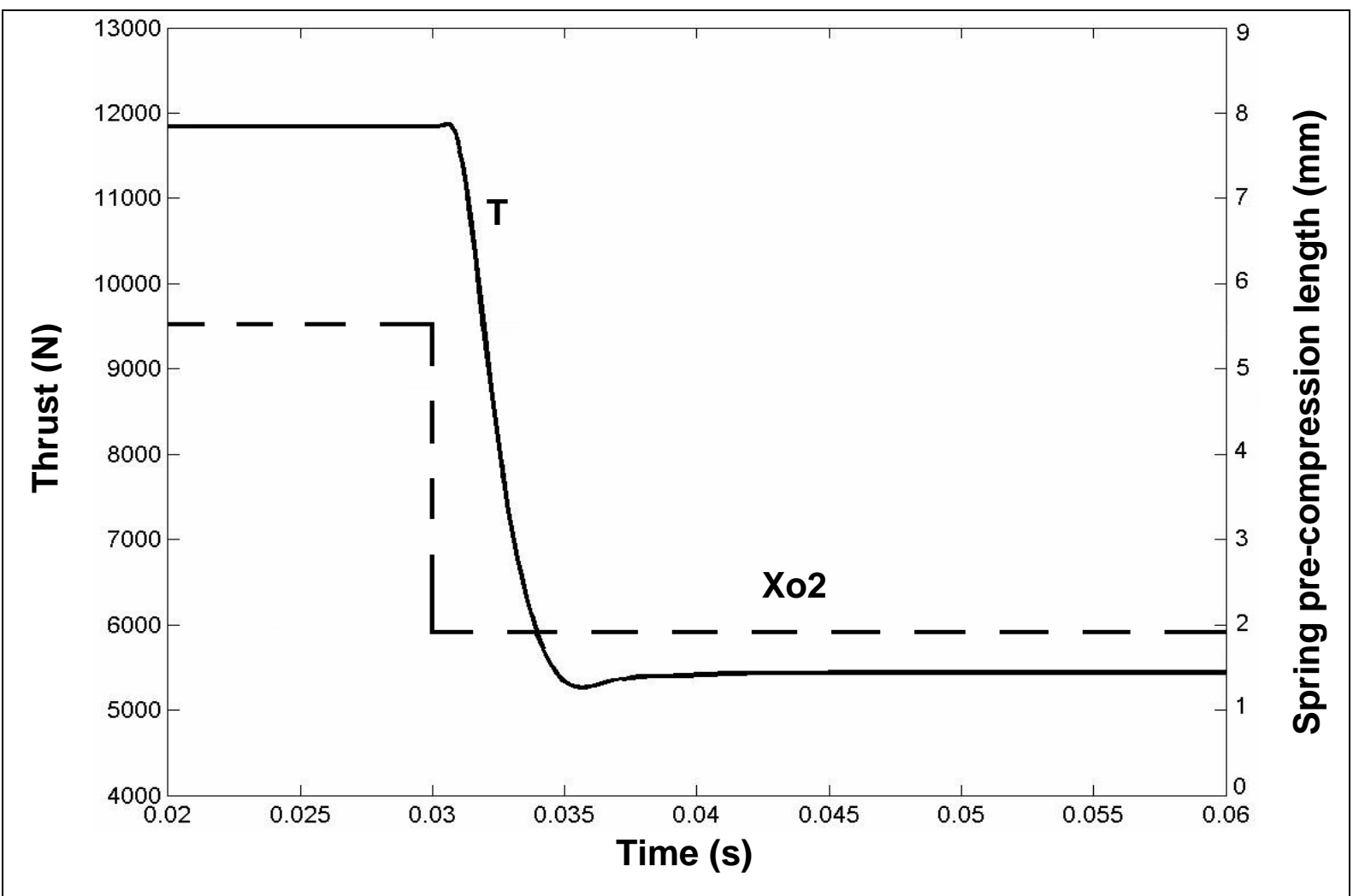

Fig.11. Thrust-time relation at a single step variation of the pre-compression distance of the thrust controller spring 


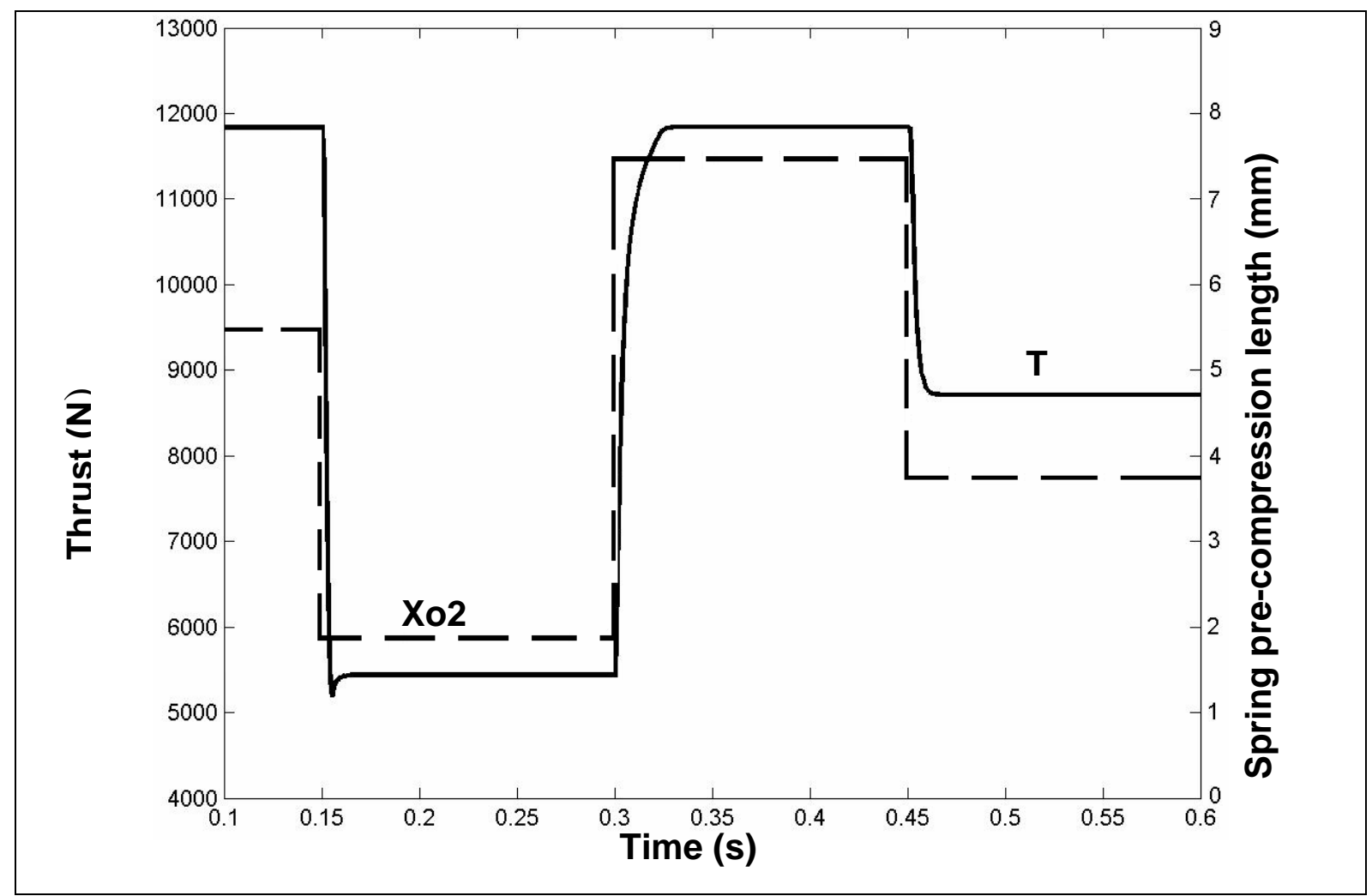

Fig.12. Thrust-time relation at a multiple step variation of the pre-compression distance of the thrust controller spring

\section{CONCLUSION}

The simulation and analysis of the behavior of the integrated hydraulic control circuit of the feeding system of a liquid propellant rocket motor and its different elements are carried out in this paper. The study is extended to evaluate the possibility of producing other controlled thrust-time relation. Empirical relations are developed based upon the manufacturer data. The models take into consideration the non-linear variations as well as the non-linear pressure-flow relations. The simulation is carried out using the SIMULINK simulation program. The following conclusions can be drawn from the study of simulation results: The non-linear behavior of the studied system is clearly noticeable. The system operates at a stable; self regulated condition during the first mode of operation. The switching from the first to second rating was found to have a slightly under damped nature of settling time within $10 \mathrm{~ms}$. The main factor determining the operating mode was found to be the pre-compression distance of the thrust controller spring. A proposal for obtaining new thrust-time relation was presented. Calculation of the transient response of the rocket while producing a sample thrust-time program demonstrated that the thrust always reaches a new steady state value corresponding to the spring pre-compression distance of the thrust controller. The developed simulation program could be applied for further analysis of feeding system performance, through the evaluation of the effects of other parameters such as the production tolerances and allowances. 


\section{REFERENCES}

1 Rabie, M. G., "Simulation and Analysis of a Pilot-Operated Hydraulic Pressure Reducer", 3rd ASAT conference, Military Technical college, Cairo, 1-6 April, 1989, pp. 371-381.

2 Rabie, M.G., and Hafez, H.E.; "Modeling by block bond graph and investigation of dynamic behavior of a hydraulic pressure reducer" , Ain Shams University, Engineering Bulletin, vol. 26, no.1, Mars 1991, pp. 429-509.

3 Margolis, D.L., and Hennings, C.; "Stability of hydraulic motion control system", Journal of Dynamic Systems, Measurements and Control, vol. 119, no. 4, pp. 605-613, December 1997.

4 Furst, R.B., and Burgess, R.M.; "Small centrifugal pumps for low-thrust rockets", J. propulsion, vol. 3, no. 3.

5 Akers, A., and Lin, S.J.; "The control of an axial piston pump using single-stage electrohydaulic servovalve", proceeding American Control Conference, vol. 3, pp.1865-1870, 1987.

6 Stepanoff, A. J.; "Stepanoff centrifugal and axial flow pumps", second edition.

7 Merrit, H. E.; "Hydraulic control system", J. W., 1968.

8 Tamer N.M. " Investigation of dynamic behavior of feeding system of liquid propellant rocker motor “ M.Sc. Thesis, Military Technical College, Cairo, 2003. 